\title{
Implementing Cloud Solutions to Optimize the Operation of District Heating Networks with Preparation for MindSphere ${ }^{\circledast}$
}

\author{
P. Sláma ${ }^{1, *}$, J. Nožička ${ }^{2}$ \\ 1 Department of Fluid Mechanics and Thermodynamics, Czech Technical University in Prague, Czech Republic \\ 2 Head of Department of Fluid Mechanics and Thermodynamics, Czech Technical University in Prague, Czech Republic
}

\begin{abstract}
The paper describes the data mining within district heating environment (physical data temperatures and flow rates) as a basis of control and optimization of a particular district heating region. The cycle is defined: the gained data transmission to a cloud solution, subsequent data formatting and their processing by applications designed within the MindSphere ${ }^{\oplus}$ environment. The particular segments of the introduced solution are: design, activation and debugging of the senders of all wireless sensors.
\end{abstract}

Keywords: control systems, district heating, MindSphere, mathematical prediction, pipeline network, heat exchanger stations

\section{Introduction}

Due to the fact that district heating systems are a critical infrastructure, a very significant effect of the described system is the preparation of tools for continuous control of the current state of the district heating system, as well as its recent operating conditions. With these means, it is possible to ensure permanent supervision from a location completely outside the heating plant, which enables automated security supervision of these systems, which significantly affect the security of the state.

The measured data are exploited for the continuous control and optimization of the district heating network operation with a permanently progressing horizon. The outputs of such optimization are recommendations for the operator aimed at the technological process upgrade through partial interventions in the technical parameters of the equipment. At the same time, the optimization algorithm ensures that the recommended interventions do not negatively affect any other operating values of the controlled heating network.

The detailed selection of the postulated measuring points in the solved heating system, including block and house transfer stations and pipelines, predates the data mining process. The introduced solution combines the conventional approaches (for measuring, control and optimizing of these systems) with the state-of-the-art innovative approaches using the cloud solutions and the complex control - and software systems, supported by the specific applications. The utilities created for the MindSphere ${ }^{\circledast}$ environment include additional boundary criteria related to the demands of with the thermal energy supplied objects, the operational economy and the external conditions of the area.

\section{Measurement of the hot water distribution of the secondary heat network}

In relation to the activity accomplishment entitled "System for on-line optimisation of the heating network operation and utilization of the potential of thermal energy 
accumulation" (within the Competence Centre of Advanced Technologies for Electricity and Heat Generation and Distribution - Project TE01020036), the thermal- and other technical parameters within the particular heating district have been measured since the end of 2017. The aim of these measurements is the acquisition of the maximum operational data from the real system operation. The particular heating district was selected from several possible locations; the selection criterion was the high variability of the operation modes of the individual energy supplied facilities. In the case of the measured hot water line there are 10 individually located supplied objects with the very different schedules of the heat supply. The residential buildings panel construction system P1.11 from the early 1980s are predominant. The other object within the district: one modern apartment house mixed concrete-brick structure since the first decade of the 21st century, one club (hall), one technical - business object and one restaurant with the irregular (through the weekend only) disco operation. The values of the thermal energy inputs of the particular buildings are shown in Table 1.

Table 1 Categories of heat energy consumption and the calculated power consumption - Line 1 starting from VVS15 (Main distributing station)

\begin{tabular}{|l|l|ll|l|}
\hline $\begin{array}{l}\text { House } \\
\text { number }\end{array}$ & $\begin{array}{l}\text { Building } \\
\text { category }\end{array}$ & \multicolumn{1}{l}{$\begin{array}{l}\text { Required } \\
\text { power input } \\
\text { forheating } \\
\text { [kW] }\end{array}$} & $\begin{array}{l}\text { Required } \\
\text { power input } \\
\text { for DHW } \\
\text { heating } \\
\text { [kW] }\end{array}$ & $\begin{array}{l}\text { Required } \\
\text { power input } \\
\text { for air } \\
\text { conditioning } \\
\text { [kW] }\end{array}$ \\
\hline 1273 & Club & - & - \\
\hline 1253 & $\begin{array}{l}\text { Shop and service } \\
\text { of garden equi- } \\
\text { pment }\end{array}$ & 70 & - & - \\
\hline 1254 & $\begin{array}{l}\text { Restaurant with } \\
\text { disco }\end{array}$ & 70 & 20 & 150 \\
\hline 1263 & Apartment block & 110 & 11 & - \\
\hline 1255 & Apartment block & 110 & 110 & - \\
\hline 1256 & Apartment block & 110 & 110 & - \\
\hline 1257 & Apartment block & 110 & 110 & - \\
\hline 1258 & Apartment block & 110 & 110 & - \\
\hline 1259 & Apartment block & 180 & 150 & - \\
\hline 1401 & Apartment block & 60 & 90 & - \\
\hline
\end{tabular}

The acquisition of data from one-year operation cycle at least at the selected hot water distribution system was the measurement goal. The part of these data gained are the winter maximum operating temperatures in the hot water supply pipelines, as well as the multi-day summer minimums of these operating temperatures. Data related to the drinking water for hygienic purposes (DHW) heating are subject of interest. DHW is prepared in the substantial part of the analysed buildings. The location of the monitored section in the town of Strakonice in southwestern Bohemia is shown in Figure 1.

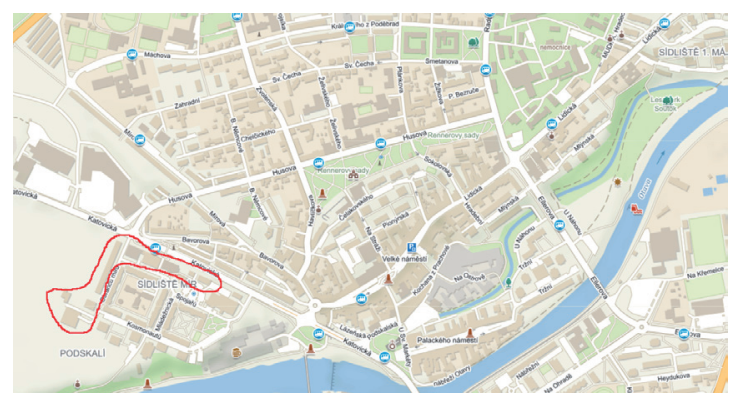

Figure 1: Location of the measured district heating region in the Strakonice town

\section{Execution of Technical Measurements}

\subsection{Pipeline network}

The buildings are supplied by the two-pipe heating network operating in the recent decades with the hot water parameters of $100 / 60{ }^{\circ} \mathrm{C}$, with the total transmission capacity of $4 \mathrm{MW}$. The basic bearing diameter of the pipeline is 2x DN150, then the pipeline network branches up to the connections $2 x$ DN40, entering into the individual house heat transfer stations (further as DPS). The only exception is the blinding of the DN100 pipe in the front of the building no. 1263. This line was originally intended for the future connection of a nearby commercial zone. This line is no longer used behind the object no. 1263. The pipe system is with the minimal altitude changes. The original hot water channel is used in several places to guide the pipeline, even if it is made of pre-insulated pipe material. This fact can be locally significant from a thermal-technical point of view.

The insulation layer of the pre-insulated pipe is made within insulation class / insulation level 1, obvious for the heat pipes in Central Europe at the turn of the 20th and 21st centuries. The layout of the entire pipeline route is shown in Figure 2. 


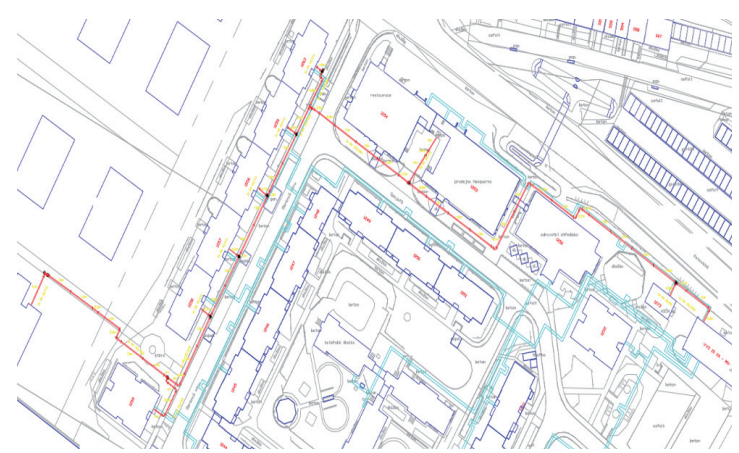

Fig. 2 Layout of pipelines - Line 1, red line, cut-out from the basic design documentation by Tenza a.s.

\subsection{Heat Exchange stations}

\subsubsection{Main transfer station (TS) VVS 15}

It is a free-standing junction heat exchanger station with operation fluids steam / water. The design parameters of the heating steam: apparent pressure $0.51 \mathrm{MPa}$, temperature $158^{\circ} \mathrm{C}$. The secondary (output) heating medium is the water with temperatures $100 / 60{ }^{\circ} \mathrm{C}$ with central equithermal control. For the Line 1, which is the subject of the measurement and optimization, the heat output of $3,880 \mathrm{~kW}$ is considered, however the entire value of 3,000 kW from it represents just the future outlook.

The supply of the Line 1 is provided by two JAD X-9.88 exchangers, $1450 \mathrm{~kW}$ each with the option for the overloading up to $2 \mathrm{MW}$.

The exchangers are governed:

qualitatively - by governing of the output temperature of the heating supply water within the range of $100-70{ }^{\circ} \mathrm{C}$. The output temperature is governed by controlled flooding of the heat exchange surface with the condensate.

quantitatively - by governing of the flow rate of the heating water at the constant temperature of $70^{\circ} \mathrm{C}$.

\subsubsection{Building (house) transfer stations}

The house transfer stations connected via the pipelines to the WV 15 are divided into three kinds of connections, depending on the heat consumption category:

1/ Pressure-dependent transfer stations designed for the heating only, no domestic hot water preparation

2/ Pressure-dependent transfer stations designed for the central heating with the domestic hot water preparation

3/ Pressure-dependent transfer stations designed for the central heating, the air heating for air conditioning and the domestic hot water preparation.

\section{The first completion into the measuring system of the heat exchanger stations}

In relation to the need of a larger amount data acquisition from the operation of the measured district heating region, the particular house heat transfer stations were completed by sensors after fully detailed assessment of the installation possibilities provided by the free slots and space inspection within the stations. These sensors enable more detailed monitoring of the temperature's course and flow rates into, out and inside stations as well. Before the mentioned installation, the individual house heat transfer stations, including the VVS 15, were monitored by the temperature sensors and calorimeters connected to the Honeywell EBI (Enterprise Buildings Integrator) control centre, version R410.2, which communicates with the Honeywell Excel 5000 sub-system controllers.

The quantity of the actually installed sensors was enlarged by temperatures - and flow rates sensors at the first stage. This enables (with deployment of the originally measured values) definition of the energy balance equations for each from the monitored circuits (external and internal) within the selected district heating region.

\section{Exploitation of the measured data for the optimization of the heating network operation}

The measured data and the design parameters of the heating network - in this particular case the monitored heating district - allow the definition of the mathematical model appropriate to this energy network. The topology of the system, the length of the individual sections, the dimensions of the pipes, the method of its installation and the insulation kind are particularly important. The model is created for two purposes: Partly for the simulator verification of the control algorithms and the continuous network parameters optimization as well as the own core parts of these algorithms. The mentioned model is inevitable premise for effective optimization as the tool of effective system interventions and their impacts monitoring.

The object-oriented modelling is exploited for the purposes of the simulator verification of the system optimization. The modelling tool Simscape is employed. The model of piping connecting the heat exchanger station with the individual heat recovering objects is shown in Figure 3. The Simulation model of the heat exchanger station 


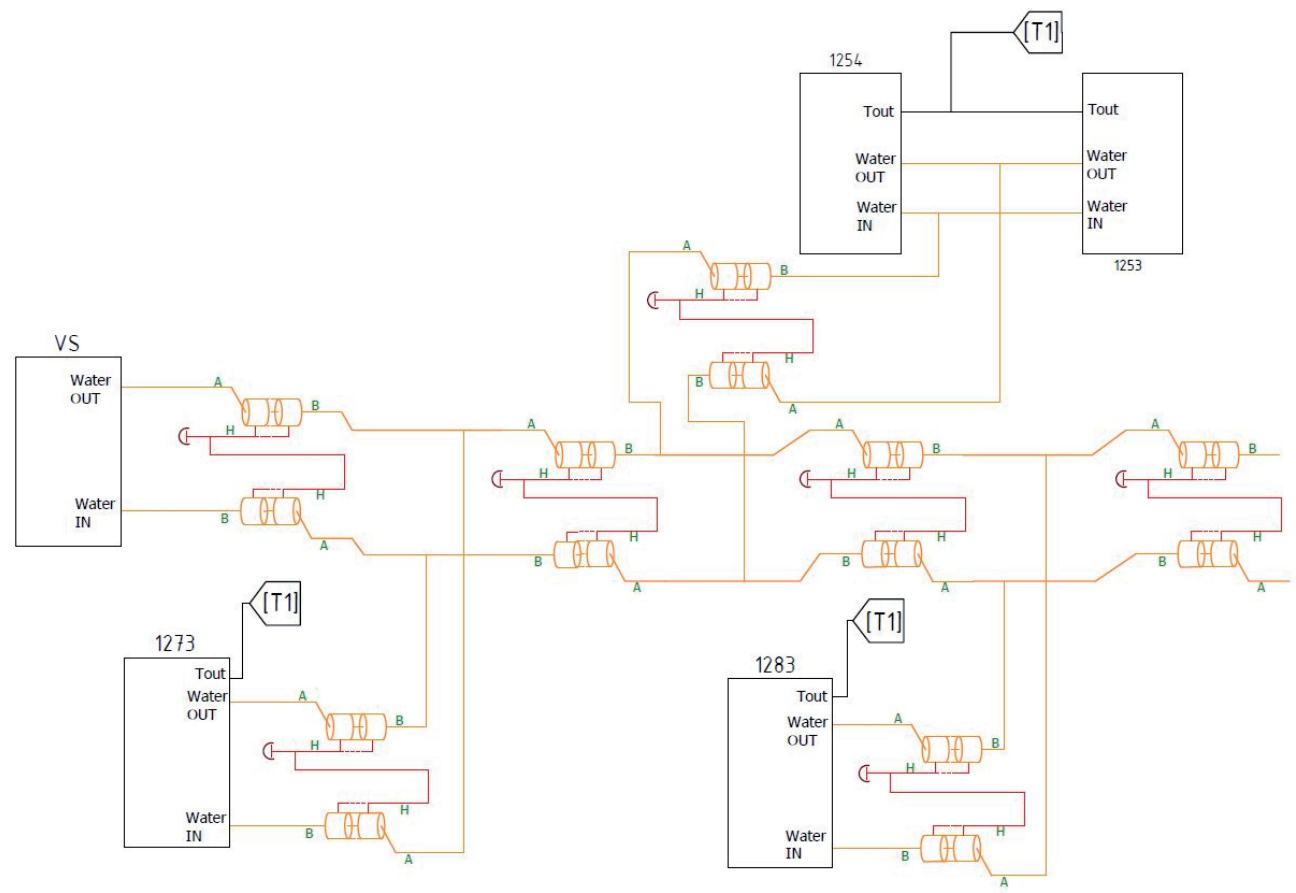

Figure 3: Simulation diagram for heating water pipelines

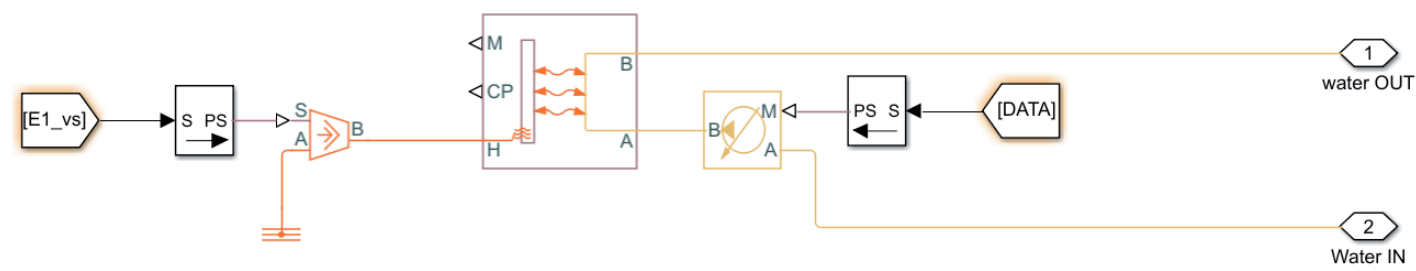

Figure 4: Simulation model of the heat exchanger station

(heat exchanger, governing unit and the pump are parts of the model) are shown in Figure 4.

Relatively simple model is needed for the continuous optimization. The work with it shall be easy and effective, the calculations has to be performed in the real time. The model based on the simplified mathematical-physical analysis, according to [1] is suitable for such purposes. As we cope with the water, i.e. a practically incompressible liquid, there is very significant difference in the dynamics of temperatures and flow rates, respectively pressures. The flow rate velocities, which roughly correspond to the speed of the temperature waves propagation, are usually in the range from c. 0.05 up to $2 \mathrm{~ms}^{-1}$, while the speed of pressure waves propagation exceeds $1000 \mathrm{~ms}^{-1}$.

The model can be accounted as the quasidynamic character, where temperatures are described by a dynamic model, mass flows or static pressures. The base of the model dynamic part is a one-dimensional flow of water through the pipe.

If we do not consider the heat accumulation into pipe material in the first step, the temperature at the pipe outlet can be expressed by an explicit relation

$$
\vartheta_{\text {lout }}(t)=\left[\vartheta_{\text {in }}(t-D)-\vartheta_{o}\right] e^{-\frac{K D}{\varrho A c_{p}}}+\vartheta_{o}
$$

where $A$ is the cross-sectional area of the pipe, $\rho$ is the density of the liquid, $c p$ is its specific heat, $v$ is the flow velocity, $\vartheta_{o}$ is the ambient temperature, $K$ is the total thermal conductivity between the running water and the ambient $\left[\mathrm{Wm}^{-1} \mathrm{~K}^{-1}\right]$ and $D$ is the transport delay. Equation (1) is derived under assumption of transport delay $D$ constant. The determination of the transport delay is probably the 
biggest issue in this otherwise very simple dynamic model, as it is variable and, depending on the flow rate and the length of the pipe $L$, the magnitude of this delay is given implicitly as

$$
\int_{t-D}^{t} v(\alpha) d \alpha=L
$$

In this relation, $\alpha$ denotes the elapsed time in the interval during which water flows through a section of pipe. The heat capacity of the pipe can be considered as concentrated at its outlet, which we get

$$
m_{a} c_{a p} \frac{d \vartheta_{o u t}(t)}{d t}=\dot{m}(t) c_{p}\left[\vartheta_{o u t}(t)-\vartheta_{\text {lout }}(t)\right]
$$

where $m a$ is the weight of the pipe material, cap is its specific heat and $\dot{m}$ is the mass flow of water. By combination of equations (3) and (1) we then get a first-order system with the transport delay, i.e. description corresponding to the general form:

$$
\theta_{\text {out }}=\frac{\boldsymbol{k}_{1} \boldsymbol{e}^{-\boldsymbol{s} \boldsymbol{D}}}{\boldsymbol{\hat { \boldsymbol { o } }}_{1} \boldsymbol{s}+1} \theta_{\text {in }}+\frac{\boldsymbol{k}_{2}}{\boldsymbol{\boldsymbol { o }}_{2} \boldsymbol{s}+1} \theta_{\boldsymbol{o}}
$$

where all $\boldsymbol{\theta}$ are temperatures, $\boldsymbol{s}$ is Laplace transform operator and

$$
\tau_{1}=\tau_{2}=\frac{\boldsymbol{m}_{a} \boldsymbol{c}_{a p}}{\dot{\boldsymbol{m}} \boldsymbol{c}_{\boldsymbol{p}}} ; \boldsymbol{k}_{1}=e^{-\frac{\boldsymbol{K} \boldsymbol{D}}{\varrho A c_{p}}} ; \boldsymbol{k}_{2}=1-e^{-\frac{K \boldsymbol{D}}{\varrho A c_{p}}}
$$

The model composed in the above way describes always just one section of pipeline. The resulting model can be obtained by combination of the partial models of the individual sections and similarly simply conceived models of pumps and exchangers. For the purposes of the optimization and prediction of the network behaviour, it is necessary to consider such model in the discretized form.

Some aspects of the cost-effective optimal operation seem to be intuitively apparent. For example: it is evident that the heat losses increase in relation to temperature rise at the energy source outlet or at the inlet of the considered subnet. However, it cannot be simply declared that the economic optimum fairly occurs at the lowest possible temperature at the heating network inlet. Deep low temperature leads to the need for the higher flow rate and thus the higher power and energy consumption of circulating pumps.
This is the reason, why the continuous optimization works with a criterion function, which characterizes the optimization goals. In the case (as introduced in this case), that the optimization of one district, but not the whole network (including the source) is performed, consideration and reduction of the heat losses and the operating costs of circulating pumps has made sense. The steady heat energy consumption from the superordinate network towards the main transfer station could be demanded at the same time. Such criterion function must be evidently supplemented by the limitation's conditions. These insure the compliance with the required temperatures in all individual house transfer stations, covering of the heat consumption etc.

The minimization of the criterion function could be always granted once with the horizon of one day (for example). The heat consumption forecast can be composed with the limited accuracy only. Therefore, it is more advantageous to repeat the optimization in each time step and always implement the first stage of the schedule only, i.e. to use the principle of declining horizon, which is the root of the predictive control based on the model.

\section{The other benefits of measurement}

The secondary benefit of the measured data and their continuous evaluation was (gained already in the first months of the supplemented measurement (1st half of the 2018)) the indication of some fittings inside of the individual PCBs, which did not perform their function properly. The indicating physical value was the cyclically increasing temperature at the cold drinking water supply into the domestic hot water exchanger in this case, even at any larger distance from this exchanger. The local investigation provided with the wrong installation of the non-return valves. This caused the opening of throttles in the closing direction as a result of the expansion of the water and the air contained in the water. The non-return valves were removed to the more gravitationally suitable positions and the described hygienically hazardous phenomenon was eliminated.

The three-way valve underflow supplying one of the supervised objects equipped with the central heating circuit caused the heat supply even at the summer time, when it was not desirable. This is another operationally significant finding. Defected fitting was adjusted and its correct function was 
checked by the test procedure after its repair.

\section{The second complement of the measuring system of the heat exchanger stations}

It was identified during the measurement, that calorimeters, belonging to the permanent equipment of VVS 15 and home heat transfer stations, albeit allow retrieval of temperatures from the internal memories, however these temperatures are averaged over operational period of 10 minutes and not the immediately measured or averaged over the required shorter period (no longer than 3 minutes). Unfortunately, the temperatures measured on the calorimeters showed deviations from the actual temperatures. This is the consequence of the calorimeter's calibration. The temperature sensors are calibrated not to the exact temperatures on the supply and return lines, but to their temperature differences, used for the heat consumption calculations, in accordance with standardized rules. It was detected also, that the data transmissions are affected by significant failures (in the size of minutes, hours, however the longest outages of several days also). The issues were related in some cases to the atmospheric disturbances surrounding the measurement area. These faults significantly affected the operation of the EBI control system and thus the transmission of the measured data. Possibilities for retrospective reconstruction of the measured, but lost data were possible in the scale of individual hours, but not the whole days.

Based on these factors, which introduce the inaccuracies and discontinuities into the data flows, it was decided to replace the temperature values transfer from the calorimeter memory by the separate temperature measurements (i.e. new measuring points) with the direct transmission to the cloud. The data formatting for the MindSphere ${ }^{\circledR}$ environment (Siemens) was initialized.

\section{Data transmission to the cloud and data processing within the MindSphere ${ }^{\circledast}$ environment}

The problem with the classic measuring systems, which are based on the robust protocols such as M-BUS or C-BUS, is in the vast majority the cumbersomeness of the entire systems. The ending parts of temperature measuring circuits are usually equipped with the resistance temperature sensors, whose information about resistance (temperature) are sent by 4-20 mA converters to the concentrators or the intermediate stages of the entire measuring system. In the most cases, the fixed time interval is set for the measurement or measuring runs continuously. The firmware- or terminal modification is time consuming obviously, or even impossible due to the manufacturer's encapsulated protocol. The continuous need for the power can be an obstacle when installing the device itself into any new places. The operation is sensitive to the power failures also. Nearby to the power supply system is located the physical (more often still metallic, sometimes optical) communication network, which is pre-designed for a specific use. Its extension is feasible to the limited scope in some cases, in other cases it is fully impossible.

Based on the mentioned factors, which introduce the inaccuracies and discontinuities into the data flows, it was decided to replace the temperature values transfers from the calorimeter's memories with the separate temperature measurements (i.e. new temperature sensors) with the direct transmission to the cloud. The data formatting for the MindSphere ${ }^{\circledR}$ environment (Siemens) was initialized. For more details, see [1].

The MindSphere ${ }^{\circledast}$ is the open operating system, developed by Siemens Co. The system provides with the data collection, storage and processing by exploitation of the system containing utilities or additionally created ones.

The purpose of the direct data transmission to the cloud is the elimination of the long-term failures in the data flow. The aim of the data storage in the MindSphere ${ }^{\circledast}$ is their availability for their further processing and evaluation within this environment.

The current development of the measuring and communication technologies clearly pushes the exploitation of up-to-date lean communication protocols and the so-called lowpower communication infrastructure. In these applications, developers try to work with such information only, where are really important and to compress the transmitted message sizes to the smallest possible files. It leads to short, simple and data-efficient transmissions (with adequate security and low data loss similarly as by the conventional networks standards). The large savings of electricity is achieved. Especially in the periods when the device does not transmit information. The sensors are in deep sleep mode with minimal energy consumption. The sensors are activated after certain 
time break, which can be controlled remotely while communication with the server.

The need of the measurement of temperatures at the heat exchangers and the transfer stations with the different measurement intervals (with the option for adjustment of the measuring interval in relation to the amount of generated data size and to energy stored in battery savings also, for example in the summer months (lower heat consumption, longer dynamics, etc.) arose within the scope of the Competence Centre of Advanced Technologies for Electricity and Heat Generation and Distribution - Project TE01020036. The particular measuring technology was selected mainly with aim for the further compatibility with the IIoT (Industrial Internet of Things) technology. This technology is the rapidly developing kinds of communication in the industrial applications.

The Figure 5. shows the anticipated reasons for the introduction of the IIOT technologies. The first "drivers" in the IIOT market focused on thermal- and electric energy dispose with feedback from the consumption end: distributed energy generation and storage, smart measuring and remote monitoring [2]. The utilities such as the thermal- or electric energy distribution are the most likely industries which will adopt the IloT technology in the mass extends. The composition of the device for communication between separate segments of a smart grid was one of the tasks of the introduced CK PTTE project. The manufacturing of the new measuring wells on PCB and VS devices was assessed as feasible. The decision for installation of 22 measuring sensors was adopted. Each sensor is equipped with the measuring development kits with the open firmware which enables modifications in relation to the reasons mentioned in the above.

Communication between the sensors and the data aggregator (cloud) proceeds via the MQTT protocol. It checks the successful data storage in the database at the same time. The server runs on the Raspberry Pi 3 platform with the Raspbian operating system. The database is of the SQL kind and the interpretation runs via the GNU visualization tool - Grafana. There is an option for the remote connection to the server in the secure way using a VPN.

It was rational to design the entire communication system based upon open source platforms for the further possibility of detailed system modifications. The option with the IloT with the MindSphere ${ }^{\circledast}$ was considered for this entire application at the same time. The system itself is of the open kind and provides with option for implementation of actual digital process transformation. The system is suited for applications on the area of preventive maintenance, energy data management, resources utilization optimization, online equipment monitoring, data collection and technology life cycle analysis and many other

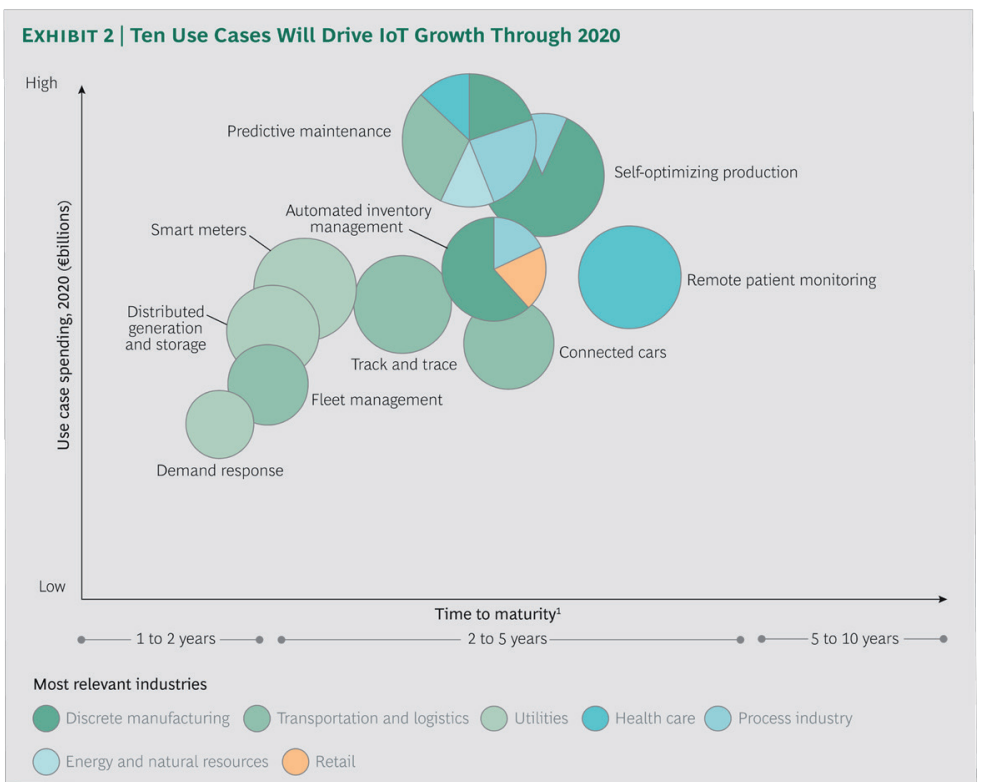

Figure 5: Assumed reasons for the introduction of loT (inc. IloT) and the industries concerned [3] 
uses. Especially the system analytical capabilities in the heating industry applications appear as very advantageous. The newly installed communication technology mounted on temperature sensors within the home heat transfer stations, as well as on VV 15 is capable to fully communicate with the MindSphere ${ }^{\oplus}$.

The system enabling supervision of the entire Line 1 operation, incl. all exchanger stations, is implemented currently in the MindSphere ${ }^{\circledast}$ environment. The supervision is secured via alerts of various weights, responding to the values of individual physical quantities, their trends, as well as to the mutual relations of these quantities, in predefined time sequences. This system is fully functional.

The graphical interpretation of the values essential for the efficient heating network operation with the option of password-protected access from any place where the connection to the Internet is available, is performed on-line.

The implementation of the above optimization tasksintotheMindSphere ${ }^{\circledR}$ environmentisthealready accomplished activity. The permanent supervision of all the transfer stations on the introduced hot water network (Figure 2) is presented in Figure 6 with the evaluation of the failure's frequency (Figure 7) and their dynamics (Figure 8) over time is already established. Another aim is the permanent determination of the optimization goal based on the previous development of the measured values and the possible prediction of these values for the near future and the ambient conditions of the managed heating network. In the discussed case the system output (in the MindSphere ${ }^{\circledR}$ environment) will be the recommended settings of the thermal network parameters performed through the proposals for changes of controlled parameters settings, such as flow rates, temperatures, etc. Such control approach is comparable with the method of permanently

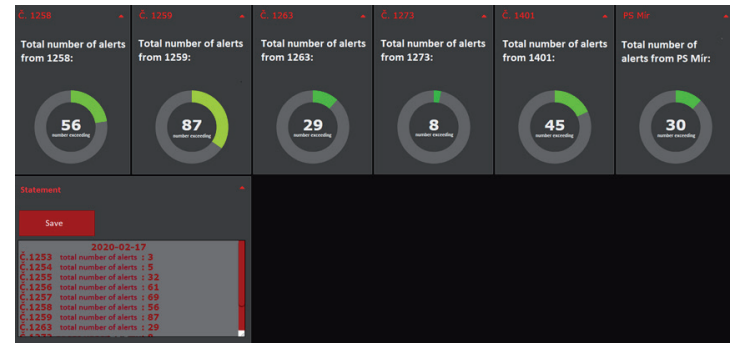

Figure 6: Error warning alerts in the operation of each of individual transfer stations for a specific day. Individual rectangles indicate individual heat exchanger stations. The number in the circle indicates the number of warning messages for a specific heat exchanger station in the last day.

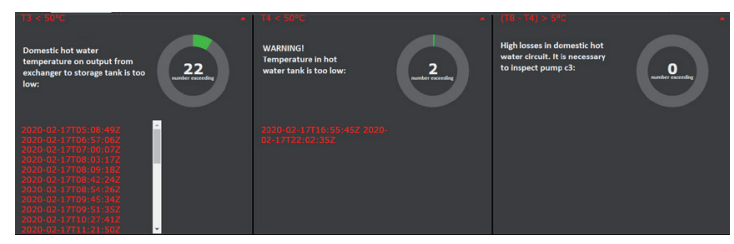

Figure 7: Description and duration of individual incorrect and inefficient operating states of hot water heating and transport for one house heat transfer station. Each rectangle shows one operating condition for this one station and information on how many times this condition was violated in the last day and when, and in what time interval, this violation occurred.

learning neural networks.

The last foreseeable step should naturally be the system for control of all individual parts of the network. This is the reason for the consideration to build the measuring and communication part on the open source and GNU platform. However, for the system control it is better to exploit the introduced IloT system MindSphere ${ }^{\circledR}$ with respect on the intervention reliability on the ending control unit, i.e. valve, heat exchanger or pump. Such solution is more robustly linked to the control technology itself. The beneficial is, that the currently installed measuring part on the introduced thermal system is fully compatible with MindSphere ${ }^{\oplus}$.

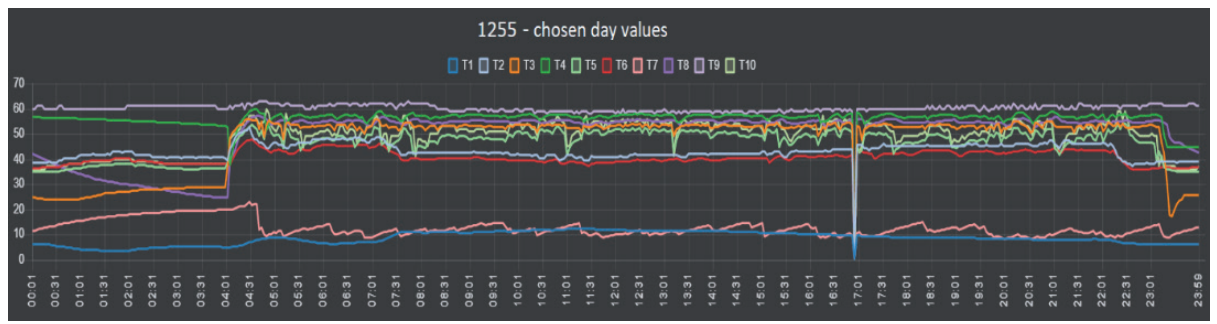

Figure 8: Progression of dynamics of operating values inside temperatures one exchanger station. This is an operating station in the house No. 1255, which is a residential house. 


\section{Conclusion}

The performed measurements and the followup measured data processing demonstrate that multiple local measurements of the physical quantities at the heat exchangers and the heat distribution of heating networks with direct data export to the cloud and its further processing in the MindSphere ${ }^{\oplus}$ are currently usable and sufficiently reliable technologies. The application in the MindSphere environment provides with the processing of the received data with respect to the required criteria. The large amount of data describing the substantial variables within the heating network, which can thus be scanned and concentrated in the cloud at the real time, shall also allow the continuous optimization of the heating network operation or its sub-district. Due to the fact that the data will be available from the deep past time segment, it is possible to create predictive models for the future heat consumption values based on such data past trends and to utilise them in optimization tasks.

\section{Acknowledgement}

The work presented in this paper has been supported by the Technology Agency of the Czech Republic (research project No.TE01020036)

\section{References}

[1] HLAVA, J.; SLÁMA, P; MUŽíK, V; Research Report No: TE01020036-V136; Technology Agency of the Czech Republic, December 2019

[2] Official documentation of the IIoT platform MindSphere ${ }^{\circledast}$ from Siemens, Available from: <https://assets.new.siemens. com/siemens/assets/public.1520420739.6fa69a2da4ac6 c37c2a224f713871ddd33925940.brozuramindsphereczk. $\mathrm{pdf}>$.

[3] HUNKE, N.; YUSUF, Z.; RüßMANN, N.; SCHMIEG, F.; BHATIA, A.; KALDRA, N.; Winning in IoT: It's All About the Business Processes, BCG. Perspectives by The Boston Consulting Group, 2017.

[4] Official documentation of the MQTT protocol, version 3.1.1. Available from: <http://docs.oasis-open.org/mqtt/mqtt/ v3.1.1/os/mqtt-v3.1.1-os.html $>$.

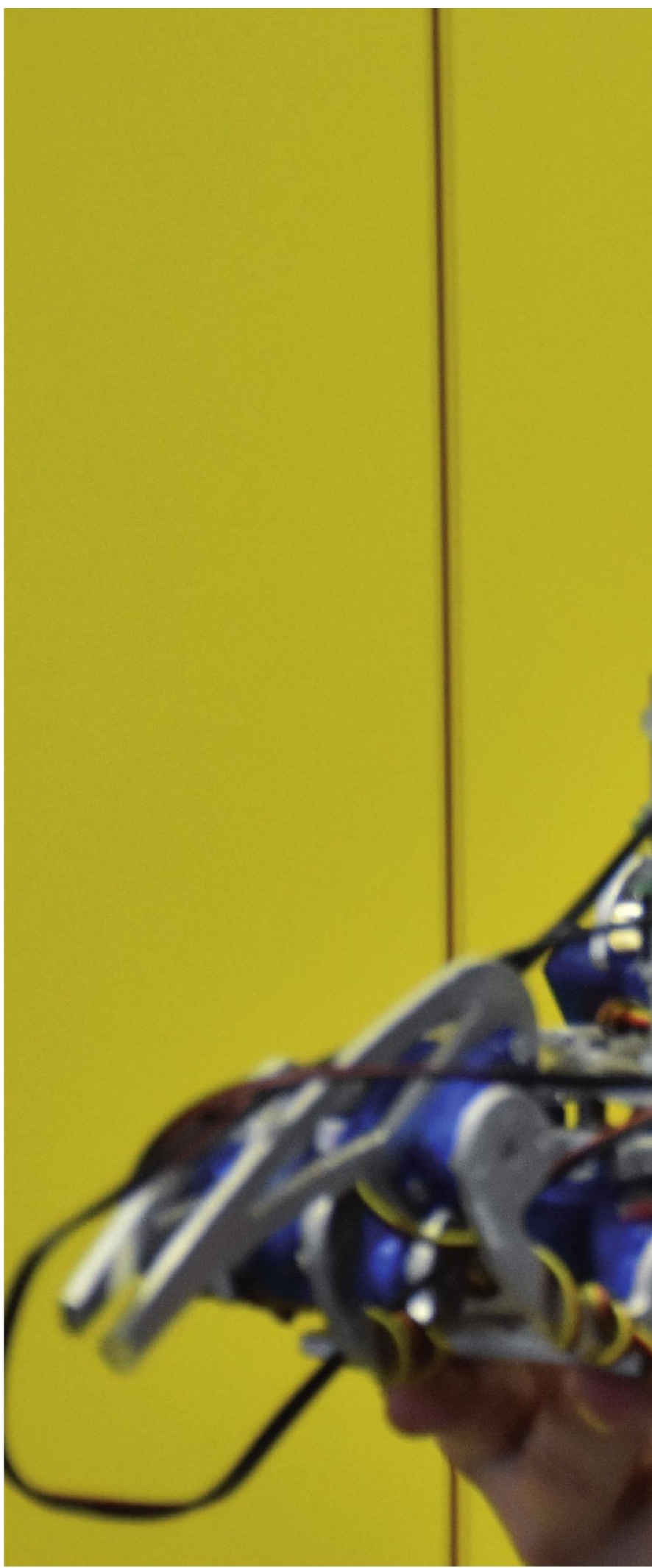

